\title{
Recovering Suppressed Resonance Frequency by Bias Lines in Reconfigurable FSS
}

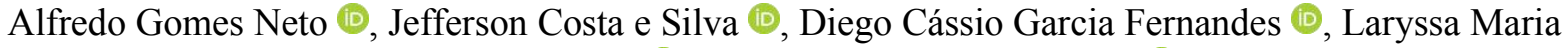 \\ de Sousa Duarte $\mathbb{P}^{\text {, }}$, Gabryel Jerônimo de Morais \\ Group of Telecommunications and Applied Electromagnetism, GTEMA \\ Federal Institute of Paraiba, IFPB, Paraiba, Brazil \\ alfredogomes@ifpb.edu.br, jefferson@ifph.edu.br,diegocassio34@gmail.com,maria.laryssa@gmail.com, \\ gabryeljmorais@gmail.com
}

\begin{abstract}
In reconfigurable frequency selective surface, FSS, bias lines can suppress a resonant frequency. This is a disadvantage, as it reduces a FSS potential application. In this paper, a procedure to recover the suppressed resonant frequency is described. A FSS based on four arms star geometry is considered and its reconfigurability is achieved by the use of varactors, for which bias lines suppress the $x$ polarization resonance. So, dipoles are added to the unit cell geometry recovering this resonance. In order to validate the proposed procedure, a reconfigurable FSS using the varactor SMV1231 ( $\left.0.466 \mathrm{pF} \leq C_{v} \leq 2.35 \mathrm{pF}\right)$ was fabricated and characterized. Numerical and measured results shown a good agreement, confirming that the FSS reconfigurability is preserved and the suppressed resonance recovered.
\end{abstract}

Index Terms - Four arms star, reconfigurable FSS, suppressed resonance, varactor.

\section{INTRODUCTION}

Frequency selective surfaces, FSS, have attracted the attention of several research groups, both for simplicity of manufacture and for the diversity of applications [1]-[3]. Dual band antennas systems [4], [5], radomes [6], [7], electromagnetically smart buildings [8]-[10] and reconfigurable antennas [3], [11] - [13] are examples of FSS applications. Basically, a FSS is composed by a periodic array of unit cells, usually engraved on a dielectric substrate, designed to be transparent in some frequency bands, while reflecting or absorbing the others. The FSS frequency response depends on the type of unit cell (patch or aperture), its geometry and periodicity, as well as substrate properties (e.g. thickness and permittivity) and polarization of the incident wave [4], [14]. Although FSS may be three-dimensional, in this paper only planar FSS are considered, that is, when the thickness of the metallization layer is much less than the wavelength at the resonant frequency [15], [16]. FSS can also present a variable frequency response, when they can be nominated reconfigurable FSS. Reconfigurable FSS are especially attractive to smart antennas applications [3], [11]-[13].

In order to obtain the FSS reconfigurability mechanical and electronic tuning have been exploited. Commonly, electronic tuning is achieved by adding active elements, such as PIN diodes, MEMS 
Journal of Microwaves, Optoelectronics and Electromagnetic Applications, Vol. 20, No. 3, September 2021 DOI: http://dx.doi.org/10.1590/2179-10742021v20i31193

switches and varactors to FSS unit cell, [3], [14], [19]-[21]. Mechanical tuning is especially attractive when no power supply is desired and, in this case, mechanical modifications (e.g., rotate, stretching, sliding, and folding) are utilized, [3], [9], [17], [18]. Both electronic and mechanical tuning can be discrete or continuous, depending on the adopted technique. In the discrete electronic tuning, when a limited number of the FSS frequency responses is required, normally PIN diodes or MEMS switches are used. For electronic tuning still, if a continuous variation of the FSS frequency response is desired, usually varactors are used.

In the reconfigurable FSS, in addition to the insertion of the active component, it is also necessary to include the bias lines, as depicted in Fig. 1, for the four arms star geometry [14]. A problem that arises is that the resonance for the polarization $x$ is practically suppressed, as can be seen in Fig. 2 . However, considering present requirements of the telecommunication systems, operating in different frequencies, retrieving this resonance may be useful, especially considering that it takes advantage of a structure that is already available, increasing its functionality.

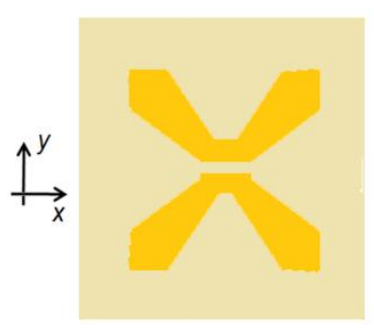

a) Geometry with gap

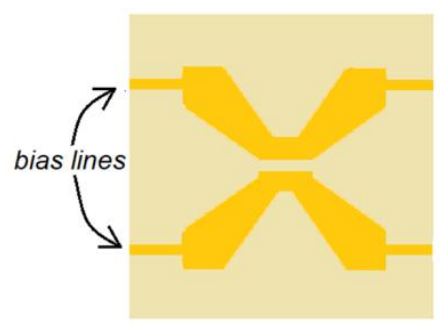

b) Geometry with gap and bias lines

Fig. 1. Four arms star geometry [14].

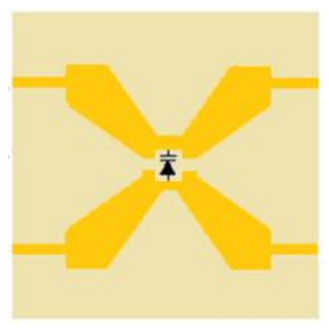

c) Varactor inserted

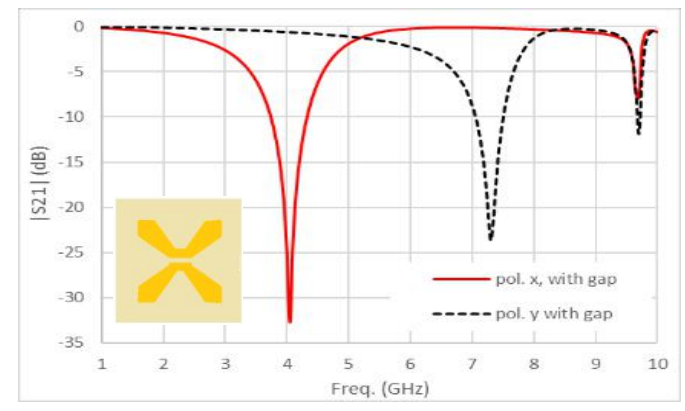

a) Frequency response without bias lines

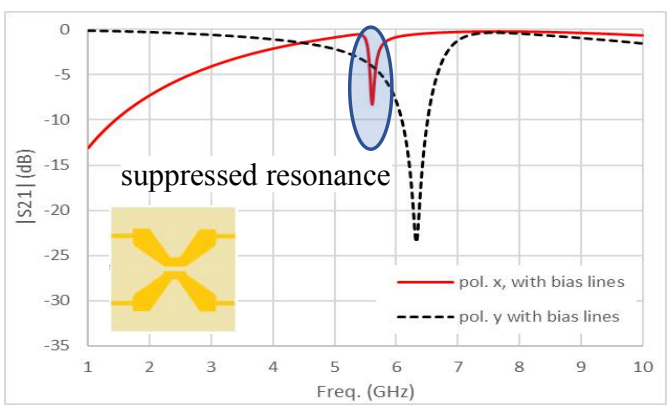

b) Frequency response with bias lines

Fig. 2. FSS frequency response with and without bias lines.

In order to recover the suppressed resonance, this paper describes the inclusion of dipoles along the $x$ direction, obtaining the respective resonance, without altering the reconfigurable behavior in the $y$ polarization. A first dipole is added between the bias lines, aligned with the switch point. Aiming to reinforce the $X$ polarization resonance, a second dipole is inserted between the four arms stars. Besides, this second dipole is dislocated to avoid a coupling with the bias lines. After this Introduction, four arms star and dipole geometry are described in Section II, which also included the varactor model. The proposed procedure to recover the suppressed resonance is detailed in Section III, including 
numerical and measured results. In Section IV, results and conclusions are summarized.

\section{FOUR ARMS STAR AND DIPOLE GEOMETRIES AND VARACTOR MODEL}

In this Section the dipole and the four arms star geometries are described, including adopted initial design equations. Furthermore, the considered varactor model is presented.

\section{A. Dipole}

Dipole is one of the simplest geometries, for which the first resonant frequency occurs when its length is approximately half wavelength, considering that the electric field and the dipole have the same direction [4].

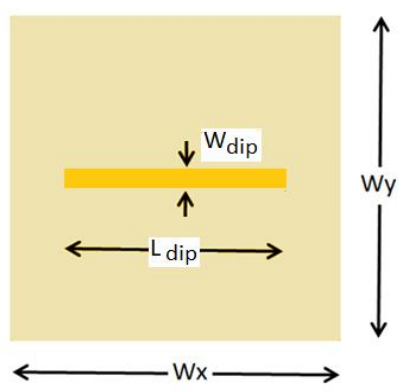

Fig. 3. Dipole geometry.

The dipole resonant frequency, $f_{\text {res-dip }}$, can be approximately determined by (1), [4], [22],

$$
f_{\text {res-dip }}(G H z)=\frac{0.3}{2 L_{\text {dip }} \sqrt{\varepsilon_{\text {ref-dip }}}},
$$

in which $\varepsilon_{r e f-d i p}$ is given by (2), [22],

$$
\varepsilon_{\text {ref-dip }}=\frac{\varepsilon_{r e f-M S}+\varepsilon_{r e f-C P W}}{2} .
$$

Where

$\varepsilon_{r e f-M S}$ is the effective dielectric constant for a microstrip, considering the microstrip width equals the dipole width, $w_{d i p}$, and a dielectric thickness $h$.

$\varepsilon_{\text {ref-CPW }}$ is the effective dielectric constant for a coplanar waveguide without ground plane, for which the distance between the center strip and the ground planes, $s$, is $10 \times h$ and the center strip width is equal to the dipole width, $w_{d i p},[23]$.

$\varepsilon_{r e f-M S}$ and $\varepsilon_{r e f-C P W}$ can be easily calculated using available software [24], [25].

\section{B. Four arms star geometry}

Four arms star geometry was introduced in [26], with very interesting characteristics, such as miniaturization and switching. In Fig. 4, the four arms star geometry is depicted and the procedure to obtain it is detailed in [19], [21]. 


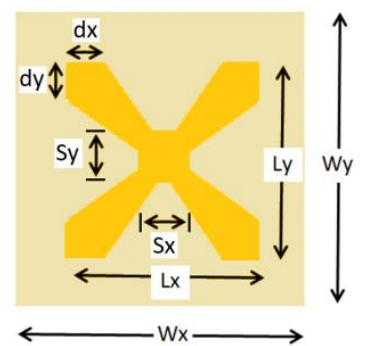

a) Geometry dimensions, without gap
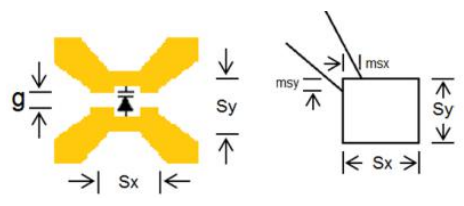

b) Gap details

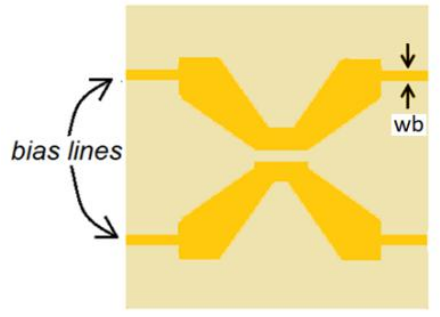

c) Geometry with gap and bias lines

Fig. 4. Four arms star geometry.

Without the gap, Fig. 4(a), the FSS resonant frequency can be estimated by (3), [14], [19], [21],

$$
\frac{0.3}{2(L x+L y)} \leq f_{\text {res }}(G H z) \leq \frac{0.3}{2\left(\sqrt{L x^{2}+L y^{2}}\right) \sqrt{\varepsilon_{\text {ref-CPW }}}},
$$

where, similar to dipole, $\varepsilon_{r e f-C P W}$ is defined as the effective dielectric constant for a coplanar waveguide without ground plane, for which the distance between the center strip and the ground planes, $s$, is $10 \times h$ and the center strip width is equal to $0.5(d x+d y)$.

With the gap and the bias lines, roughly speaking, the resonant frequency, $f_{\text {res-gb }}$, can be estimated by (4).

$$
1.5 f_{\text {res }} \leq f_{\text {res-gb }} \leq 2.0 f_{\text {res }},
$$

Despite the resonant frequencies obtained by (1)-(4) are not exact, these equations are useful as a first step for a numerical optimization.

\section{Varactor model}

The varactor is a PN junction diode that is specially designed to operate in the reverse-bias electrical condition. It takes advantage of the junction capacitance, that is increased as much as possible in the manufacturing process, and can be controlled by the reverse bias voltage [27]. A more accurate varactor model includes, besides the variable capacitance, inductances and resistances [28], Fig. 5. However, in many applications a simplified model, considering only the variable capacitance, can be adopted. A detailed modeling of the resonant behavior of a continuously reconfigurable FSS based on four arms star geometry, using the varactor as active component, is reported in [14].

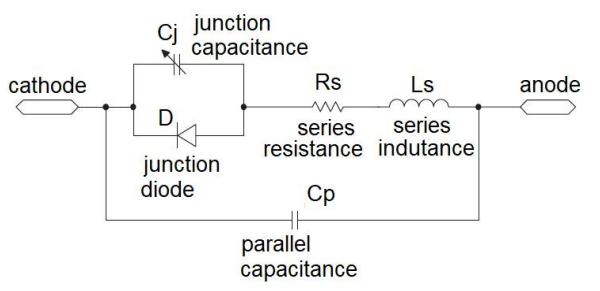

a) Detailed model

Fig. 5. Varactor models.

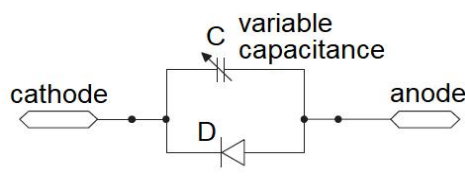

b) Variable capacitance model

In this paper the varactor SMV1231-079LF was used and its capacitance versus reverse voltage curve is presented in Fig. 6, [29]. 

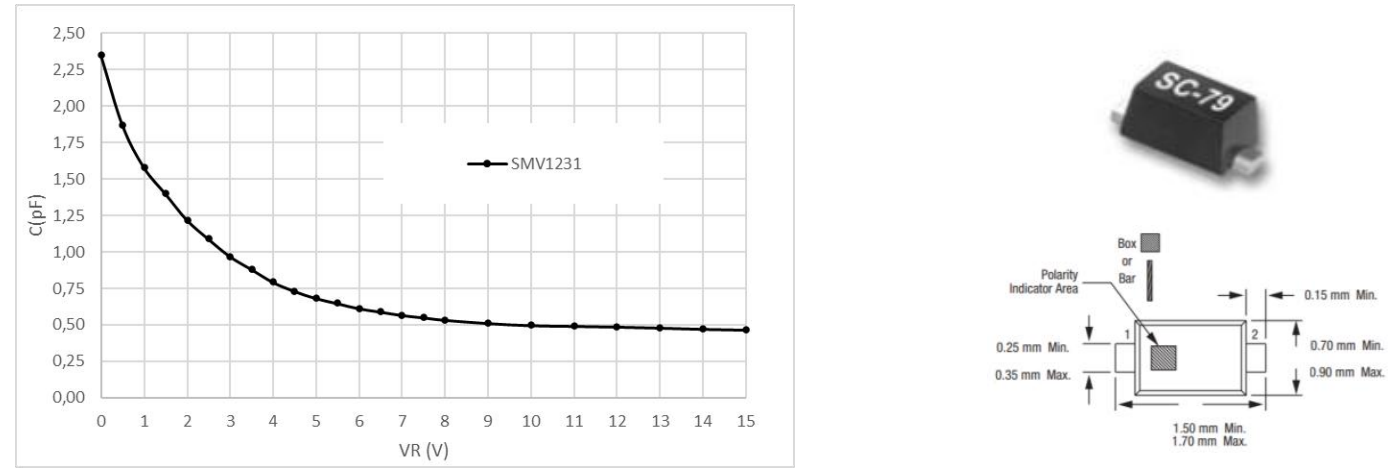

Fig. 6. Varactor SMV1231-079LF, Capacitance $(\mathrm{pF}) \times$ Reverse voltage $(\mathrm{V})$ curve [29].

In the next Section, in order to recover the suppressed resonance, the use of previously described geometries and the varactor model are detailed, including numerical and measured results.

\section{RECOVERING THE SUPPRESSED RESONANCE}

In order to verify the proposed design procedure, numerical and measured results were obtained. Numerical results were achieved using the commercial software ANSYS HFSS, and, unless specified, the incidence is considered normal to the FSS. Measured results were acquired at the GTEMA/IFPB microwave measurements laboratory using an Agilent E5071C two ports network analyzer, two double ridge horn antennas and a measurement window as shown in Fig. 7. A low-cost fiber-glass substrate (FR-4, $\varepsilon_{r}=4.4$, loss tangent 0.02 , thickness $1.0 \mathrm{~mm}$ ) was employed.

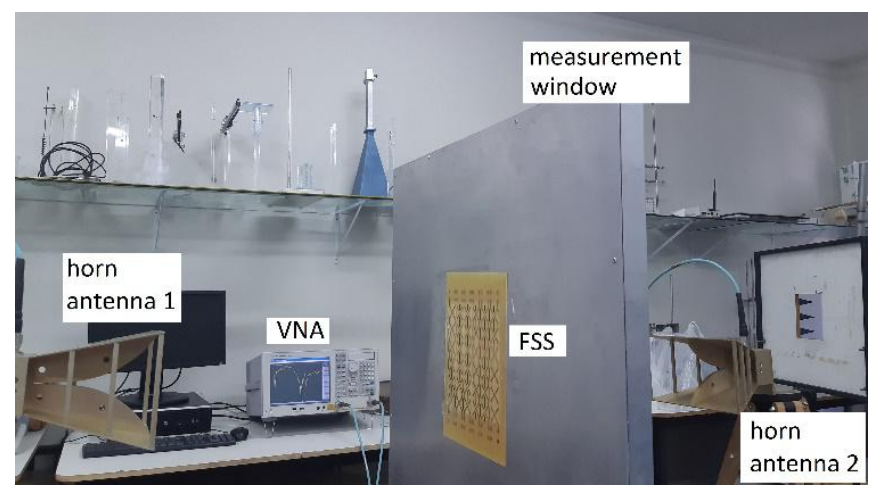

Fig. 7. Measurement setup.

As a first step, a FSS based on the four arms star geometry, Fig. 4(a), was designed with the following unit cell dimensions: $W x=W y=32 \mathrm{~mm}, L x=L y=26 \mathrm{~mm}, S x=S y=4 \mathrm{~mm}, d x=$ $d y=3 \mathrm{~mm}, m s x=m s y=1 \mathrm{~mm}$. It was numerically characterized and its frequency response is shown in Fig. 8. As expected, the frequency responses are the same for $x$ and $y$ polarizations. The first resonant frequency is $f_{\text {res }}=3.40 \mathrm{GHz}$, in accordance with (3), $2.88 \mathrm{GHz} \leq f_{\text {res }} \leq 3.52 \mathrm{GHz}$. 

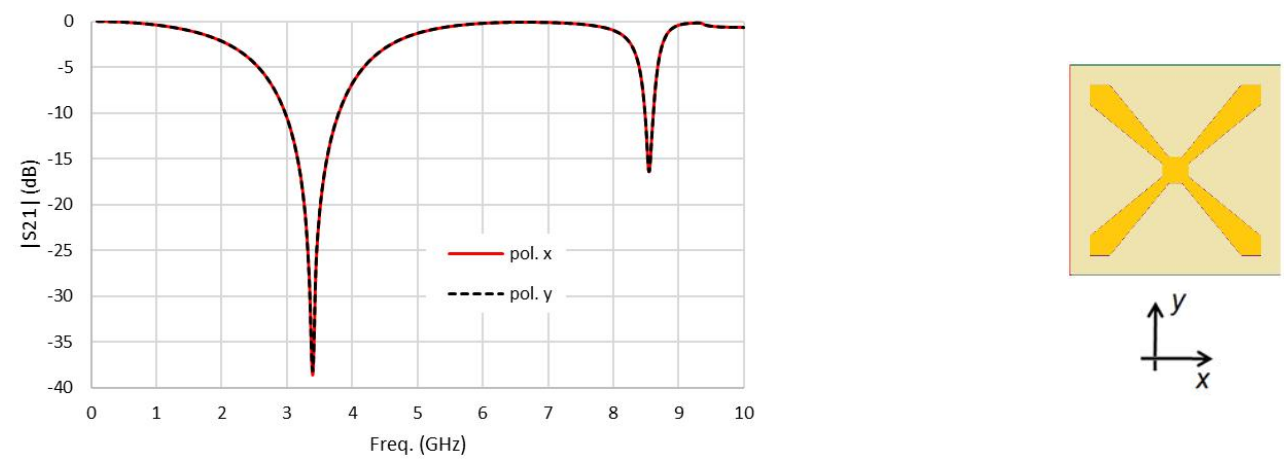

Fig. 8. FSS frequency response - Four arms star geometry.

In the next step, the gap and the bias lines were introduced ( $g=1 \mathrm{~mm}, w b=1.5 \mathrm{~mm}$ ), Fig. 4(c), and the obtained frequency response is presented in Fig. 9. The FSS became polarization dependent, with band pass and band stop characteristics, for $x$ and $y$ polarizations, respectively. For the $y$ polarization, the resonant frequency is $f_{r e s-g b}=5.75 \mathrm{GHz}$, in consonance with (4), $5.10 \mathrm{GHz} \leq$ $f_{r e s-g b} \leq 6.80 \mathrm{GHz}$. For $x$ polarization the band stop resonance was practically suppressed.
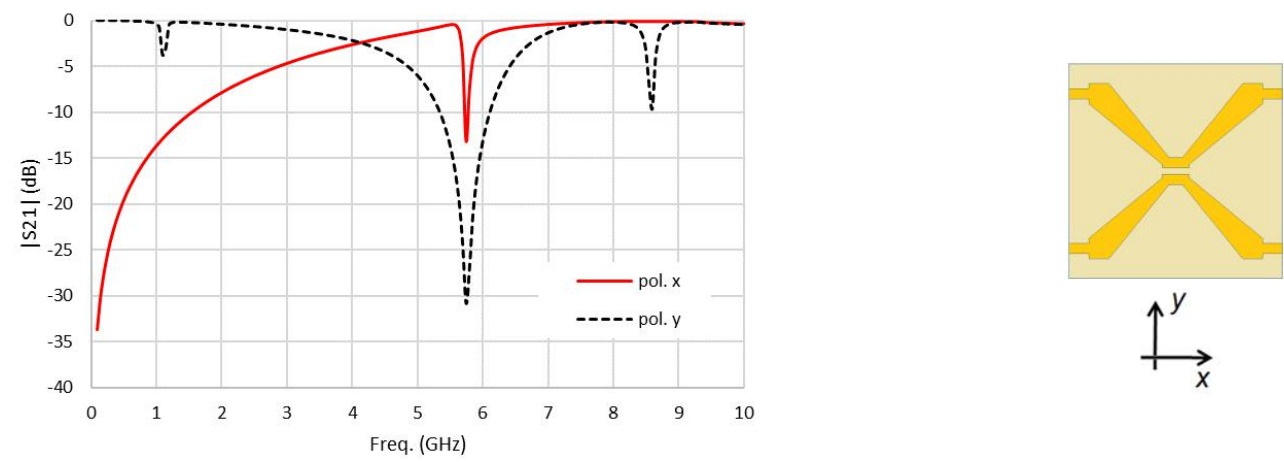

Fig. 9. FSS frequency response - Four arms star geometry with gap and bias lines.

In the dipole specification, initially it is considered a single dipole, Fig. 10(a), with $L_{d i p}=20 \mathrm{~mm}$ and $w_{\text {dip }}=2 \mathrm{~mm}$. Then, a second dipole is placed in the unit cell, Fig. 10(b), Fig. 10(c). Note that this new dipole is placed in parallel with the existing dipole, but displaced. Fig. 11 shows the frequency response for the single dipole and with the second dipole. As for the $y$ polarization no resonant frequency is observed, results are presented for the $x$ polarization only. For the single dipole, the resonant frequency determined using (1) is $4.86 \mathrm{GHz}$, a good result when compared to the numerical result, $5.20 \mathrm{GHz}$. With the second dipole, the resonant frequency, obtained numerically, is $5.75 \mathrm{GHz}$. Observe that the dipole resonant frequency can be easily adjusted, which is interesting in the FSS design. 


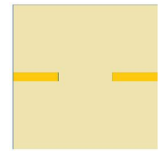

a) Single dipole

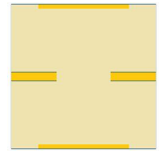

b) Second dipole placed

Fig. 10. FSS unit cell with dipoles.

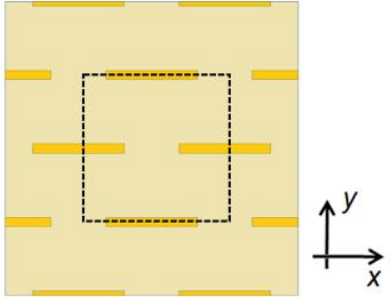

c) FSS unit cell
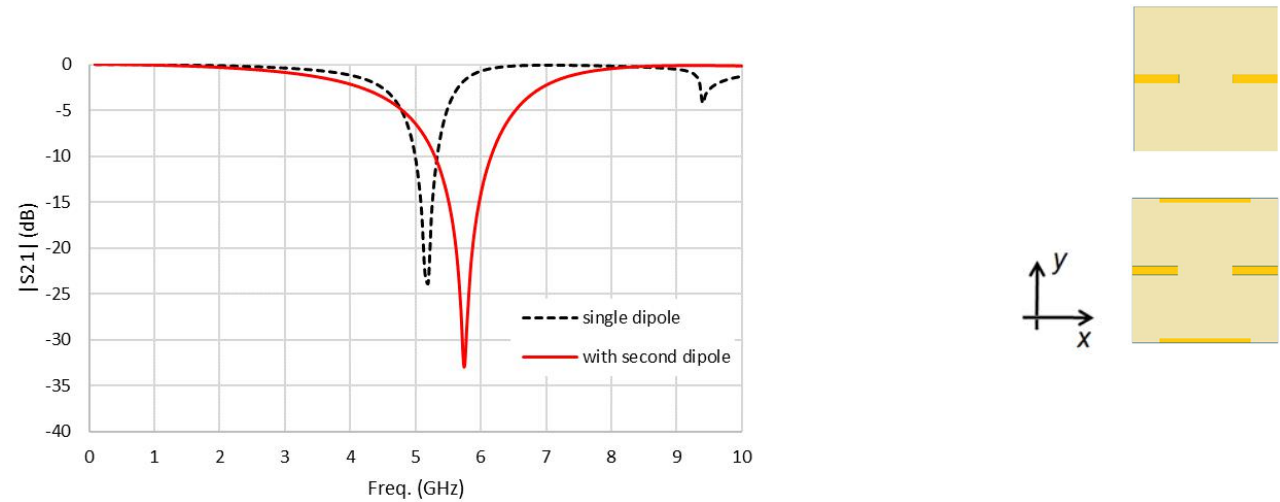

Fig. 11. FSS frequency response - Dipoles, $x$ polarization only.

Then, four arms star and dipole geometries are superposed. In this case, a FSS prototype was fabricated with the previously described unit cell dimensions. The whole FSS has $6 \times 6$ unit cells, arranged in 6 lines, with $192 \mathrm{~mm} \times 192 \mathrm{~mm}$, Fig. 12. Despite the cell lines could be individually controlled, in this work they were connected and controlled all together. The FSS dimensions are summarized in Table I. The frequency responses for the FSS prototype, without the insertion of the varactor, polarizations $x$ and $y$ are shown in Fig. 13 and Fig. 14, respectively, verifying a good agreement between numerical and measured results.

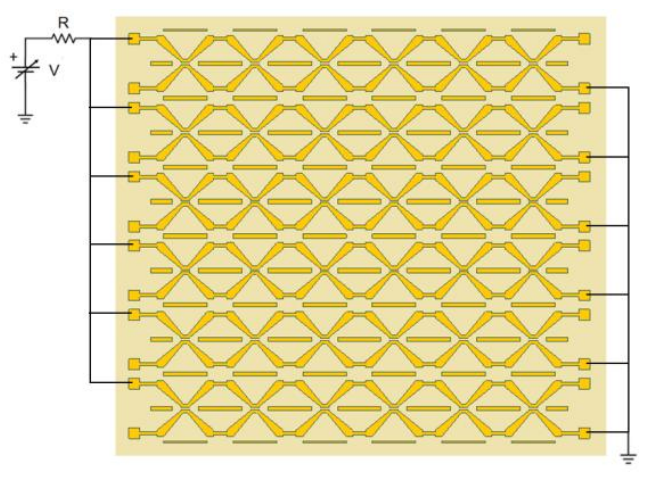

a) FSS drawing

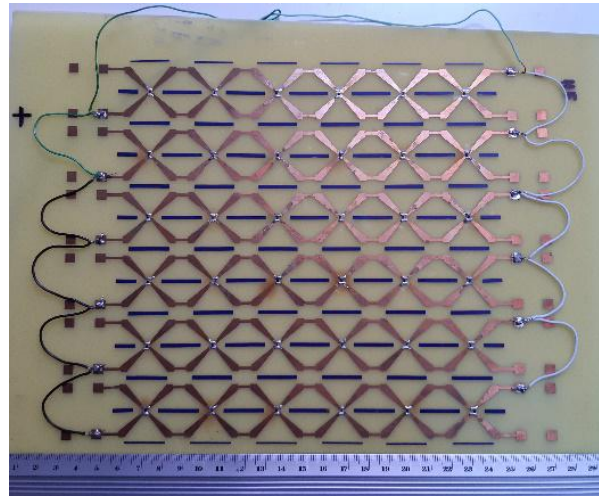

b) Fabricated prototype
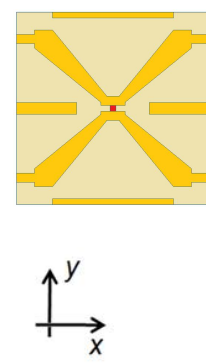

c) Unit cell

Fig. 12. FSS prototype.

TABLE I. FSS DIMENSIONS

\begin{tabular}{ccc}
\hline$W x=W y$ & $S x=S y=4 m m$ & $w b$ \\
\hline$L x=L y$ & $m s x=m s y=$ & $L_{\text {dip }}=$ \\
\hline$d x=d y=3 \mathrm{~mm}$ & $g=1 \mathrm{~mm}$ & $w_{\text {dip }}$ \\
\hline
\end{tabular}


Journal of Microwaves, Optoelectronics and Electromagnetic Applications, Vol. 20, No. 3, September 2021 DOI: http://dx.doi.org/10.1590/2179-10742021v20i31193

For $\chi$ polarization the measured resonant frequency is $6.00 \mathrm{GHz}$, not so different from the numerical value, $5.70 \mathrm{GHz}$. This resonant frequency is practically the same for the dipoles (5.75 $\mathrm{G} \mathrm{Hz}$ ), recovering the resonance for the $x$ polarization. Furthermore, the resonant frequency can be adjusted according to the dipole length and the resonance around $7.0 \mathrm{GHz}$ can be removed.

For $y$ polarization the measured resonant frequency is $6.13 \mathrm{GHz}$, a difference of $7.5 \%$, when compared to the numerical value, $5.75 \mathrm{GHz}$. Two main factors can be related to the observed differences between measured and numerical values: fabrications process limits and dielectric constant value, assumed as 4.4, which can vary from a FR-4 sheet to other.
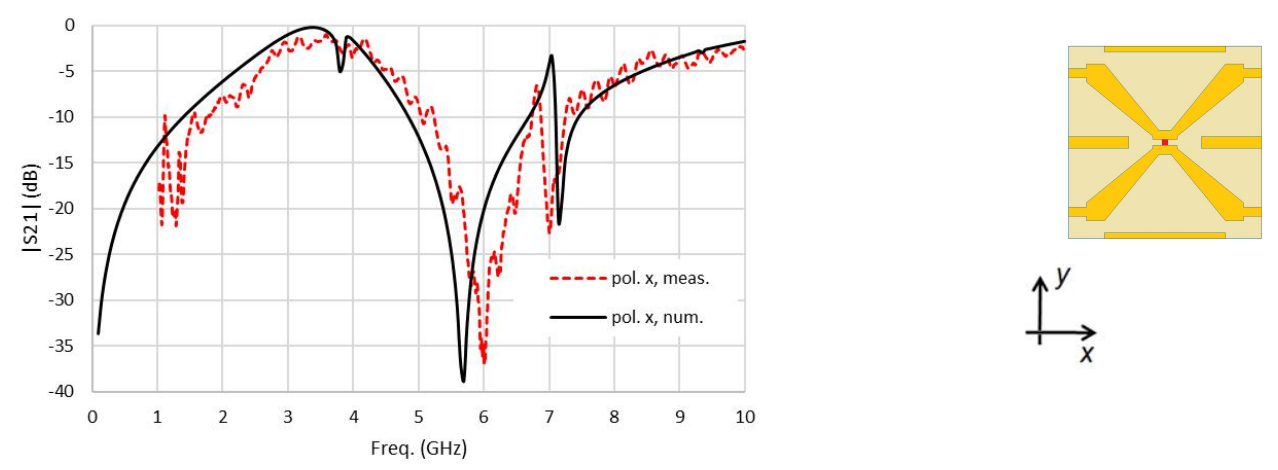

Fig. 13. FSS frequency response, without varactor insertion $-x$ polarization.
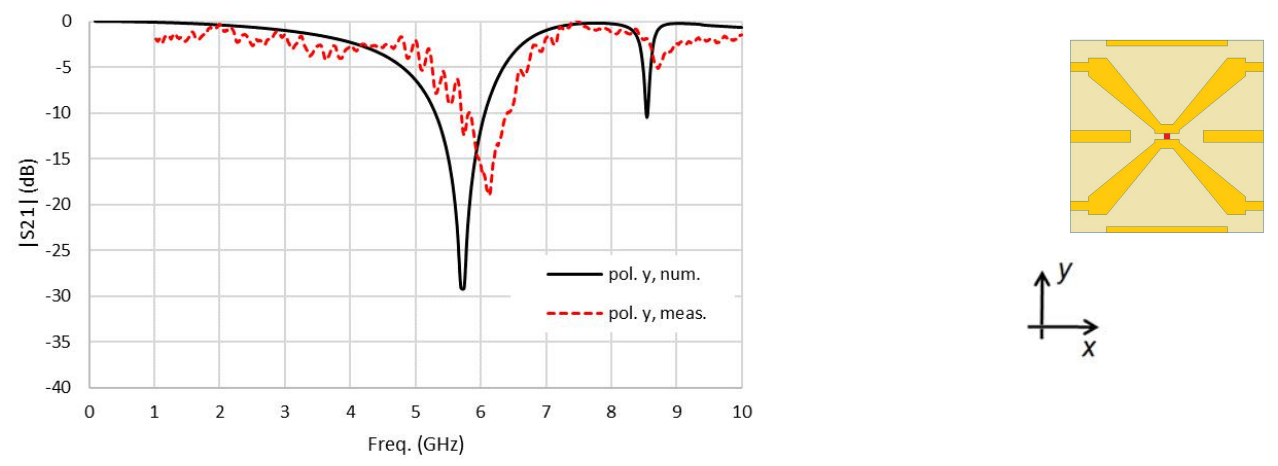

Fig. 14. FSS frequency response, without varactor insertion $-y$ polarization.

With the varactor insertion, an additional variable capacitance is introduced. The capacitance value can be estimated from the curve shown in Fig. 6, and the resonant behavior modeling is detailed in [14]. For three different varactor capacitance values, frequency responses are presented in Fig. 15 and in Fig. 16, $x$ and $y$ polarizations, respectively.

For $x$ polarization, Fig. 15, numerical results are independent of the varactor capacitance value, then only one result is shown. A good agreement between numerical and measured results is verified, confirming that $\mathrm{X}$ polarization resonant frequency is recovered.

In Fig. 16, for $y$ polarization, it is observed the variation of the frequency response with the 
Journal of Microwaves, Optoelectronics and Electromagnetic Applications, Vol. 20, No. 3, September 2021 DOI: http://dx.doi.org/10.1590/2179-10742021v20i31193

varactor capacitance. Therefore, the added dipoles did not affect the FSS reconfigurable behavior.
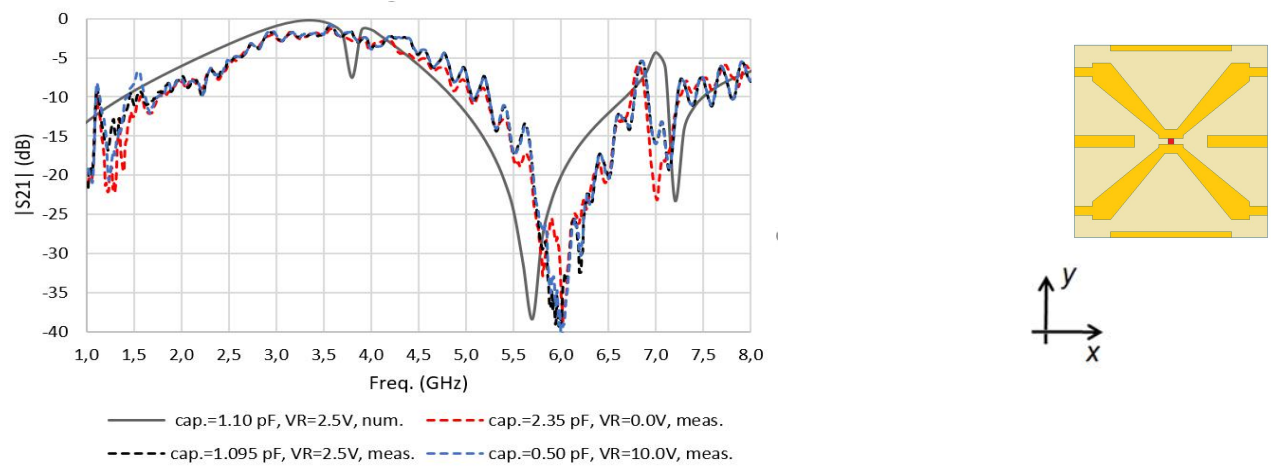

Fig. 15. FSS frequency response, with varactor insertion $-x$ polarization.
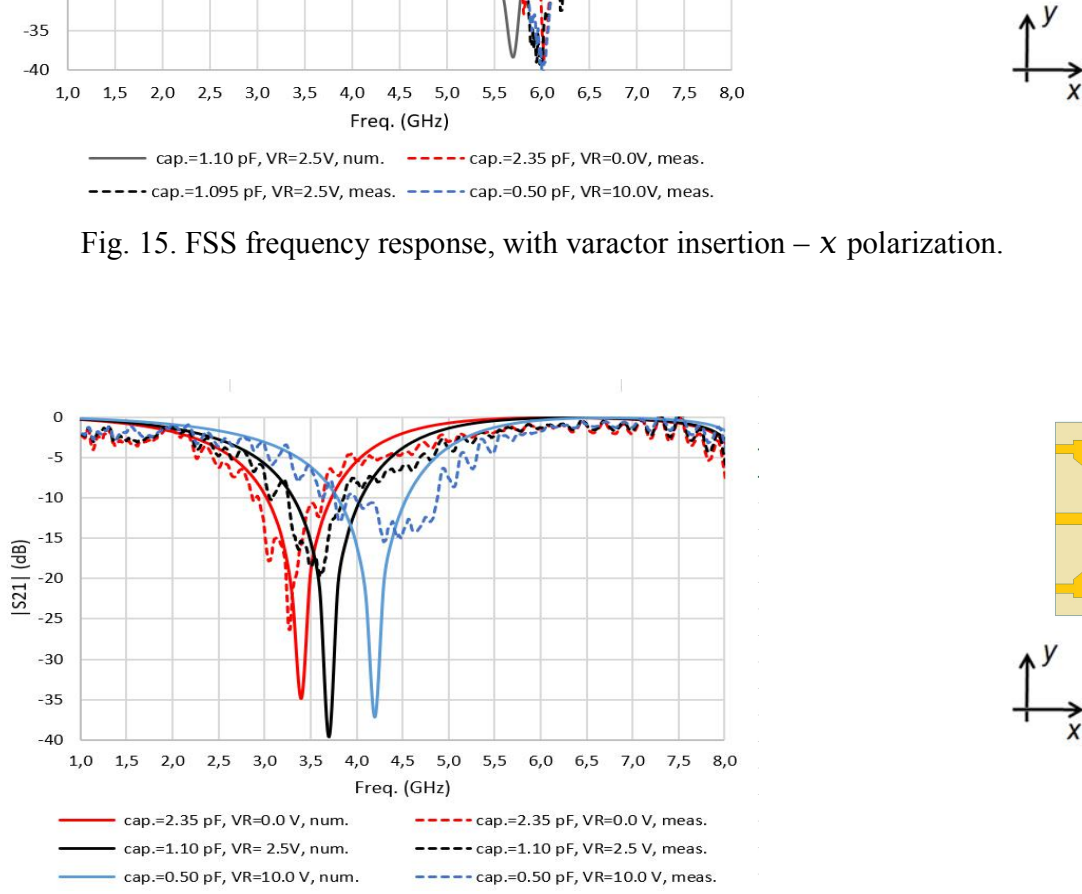

Fig. 16. FSS frequency response, with varactor insertion $-y$ polarization.

In Fig. 17 it is shown the resonant frequency as a function of the varactor reverse voltage, with a good agreement between numerical and measured results. For $x$ polarization, the recovered resonance remains practically constant. For $y$ polarization a resonance variation from $3.30 \mathrm{GHz}$ to $4.46 \mathrm{GHz}$, and bandwidth of $1.16 \mathrm{GHz}$ was achieved. These are interesting values for antennas applications.
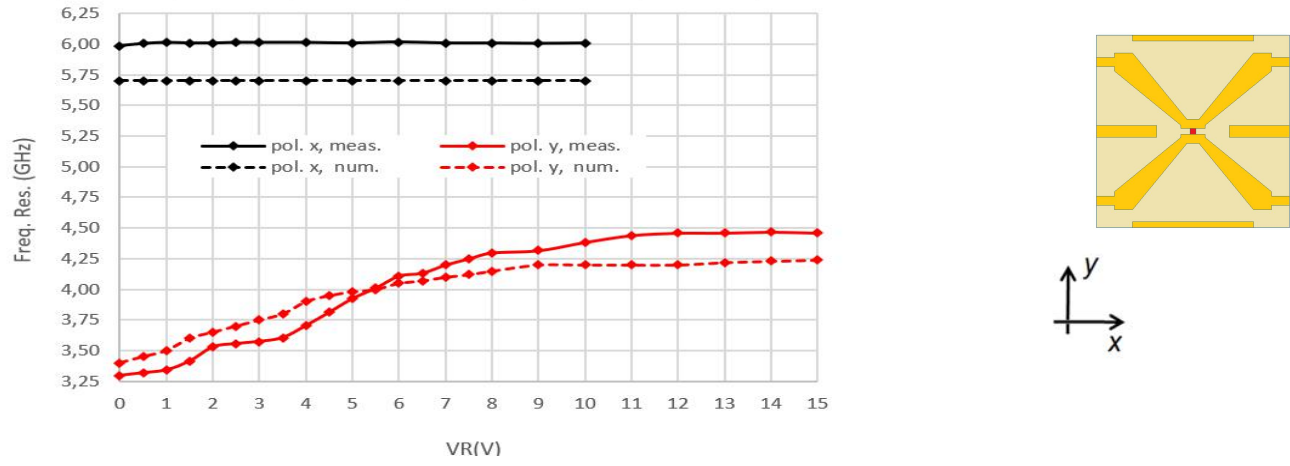

Fig. 17. Resonant frequency $(\mathrm{GHz}) \times$ Reverse voltage $(\mathrm{V})$. 
Journal of Microwaves, Optoelectronics and Electromagnetic Applications, Vol. 20, No. 3, September 2021 DOI: http://dx.doi.org/10.1590/2179-10742021v20i31193

\section{CONCLUSIONS}

As bias lines in reconfigurable FSS can suppress a resonant frequency, a procedure to recover this suppressed resonance was described in this paper. Considering the four arms star geometry, basically, dipoles are added to the original reconfigurable FSS unit cell. Initial design equations for four arms star and dipole geometries were presented, as well as the varactor model. Each geometry was numerically characterized and the results obtained indicated the validity of the initial design equations. A reconfigurable FSS prototype was fabricated with the composed geometry (four arms star and dipoles), and, without the varactor insertion, numerical and measured results confirmed that the respective geometry resonances are maintained.

The SMV1231 varactor $\left(0.466 \mathrm{pF} \leq C_{v} \leq 2.35 \mathrm{pF}\right)$ was implemented and it was verified that the FSS reconfigurability was not affected by the dipoles, achieving, for $y$ polarization, a variation from $3.30 \mathrm{GHz}$ to $4.46 \mathrm{GHz}$, a bandwidth of $1.16 \mathrm{GHz}$, an interesting value for many telecommunication applications. Furthermore, for $x$ polarization, the recovered resonant frequency remains practically unchanged (5.75 $\mathrm{GHz}$ numerical/6.00 $\mathrm{GHz}$ measured). In this way, the proposed FSS shown two distinct frequency response: a reconfigurable, $y$ polarization, and the other fixed, $x$ polarization. It must be highlighted that the $x$ polarization resonant frequency can be adjusted according the dipole length, a flexible design variable. Finally, it is possible to say that the objectives have been achieved, and a procedure has been described to recover the resonance suppressed by the bias lines.

\section{ACKNOWLEDGMENT}

The authors would like to thank to Leanne Vincent, from Skyworks, by the provided varactors. This work was supported in part by the CNPq (Project 407028/2016-1) and CAPES, Brazilian Federal Agencies, and by the IFPB, Federal Institute of Paraíba (PPGEE, PRPIPG 01/2019).

\section{REFERENCES}

[1] K. Katoch, N. Jaglan and S. D. Gupta, "A Review on frequency selective surfaces and its applications," 2019 International Conference on Signal Processing and Communication (ICSC), NOIDA, India, 2019, pp. 75-81.

[2] Rana Sadaf Anwar, Lingfeng Mao and Huansheng Ning, "Frequency selective surfaces: a review," Applied Sciences, vol. 8, no. 9: 1689, Sep. 2018.

[3] Ravi Panwar, Jung Ryul Lee, "Progress in frequency selective surface-based smart electromagnetic structures: a critical review," Aerospace Science and Technology, vol. 66, pp. 216-234, 2017.

[4] B. A. Munk, Frequency Selective Surfaces - Theory and Design, New York: Wiley, 2000.

[5] T. H. Brandão, H.R.D. Filgueiras, A. A. C. Alves, F. Scotti, S. Melo, A. Bogoni, Arismar Cerqueira S. Jr., "Dual-band system composed by a photonics-based radar and a focal-point/cassegrain parabolic antenna,", Journal of Microwaves, Optoelectronics and Electromagnetic Applications, vol. 17, no. 4, December 2018.

[6] Z. Xiaohong, L. Xiaochun, S. Shining, P. Xiaoyu and D. Jinshan, "A Hybrid structure design of broadband band-pass MEFSS and FSS," 2020 9th Asia-Pacific Conference on Antennas and Propagation (APCAP), Xiamen, China, 2020, pp. 1-2.

[7] N. Liu, X. Sheng, C. Zhang and D. Guo, "Design of dual-band composite radome wall with high angular stability using frequency selective surface," IEEE Access, vol. 7, pp. 123393-123401, 2019.

[8] S. Cho, I. Lee and I. Hong, "Frequency selective film design for building walls for blocking wireless LAN signal," 2018 International Symposium on Antennas and Propagation (ISAP), Busan, Korea (South), 2018, pp. 1-2.

Brazilian Microwave and Optoelectronics Society-SBMO received 4 Feb 2021; for review 10 Feb 2021; accepted 18 May 2021

$\begin{array}{lllll}\text { Brazilian Society of Electromagnetism-SBMag } & \text { (C) } 2021 \text { SBMO/SBMag } & \text { (cc) BY } & \text { ISSN 2179-1074 }\end{array}$ 
[9] S. Abirami B, E. Florence Sundarsingh and V. S. Ramalingam, "Mechanically reconfigurable frequency selective surface for RF shielding in indoor wireless environment," IEEE Transactions on Electromagnetic Compatibility, vol. 62, no. 6, pp. 2643-2646, Dec. 2020.

[10] M. Cerveny, J. M. Rigelsford and R. J. Langley, "A sparse FSS for control of radio coverage in buildings," 2016 IEEE International Symposium on Antennas and Propagation (APSURSI), Fajardo, 2016, pp. 1449-1450.

[11] A. Kesavan, M. Mantash, J. Zaid and T. A. Denidni, "A Dual-plane beam-sweeping millimeter-wave antenna using reconfigurable frequency selective surfaces," IEEE Antennas and Wireless Propagation Letters, vol. 17, no. 10, pp. 1832-1836, Oct. 2018.

[12] M. M. Leingthone and N. Hakem, "Implementation of a pattern-reconfigurable antenna for modern wireless sensor network applications," 2019 IEEE International Symposium on Antennas and Propagation and USNC-URSI Radio Science Meeting, Atlanta, GA, USA, 2019, pp. 683-684.

[13] L. Han, G. Cheng, G. Han, R. Ma and W. Zhang, "Electronically beam-steering antenna with active frequency-selective surface," IEEE Antennas and Wireless Propagation Letters, vol. 18, no. 1, pp. 108-112, Jan. 2019.

[14] A. Gomes Neto, J. C. Silva, A. Gomes Barboza, I. Barbosa Grécia Coutinho, M. de Oliveira Alencar, M. Correia de Andrade, "Modeling the resonant behavior of continuously reconfigurable FSS based on four arms star geometry," Journal of Microwaves, Optoelectronics and Electromagnetic Applications (JMOe), vol. 19, no. 3, pp. 415-427, Aug. 2020.

[15] W. Hu, M. Jia, Y. Dong, X. Qian, Y. Yang and X. He, "3D ultra-wideband high selective bandpass FSS," 2019 IEEE MTT-S International Wireless Symposium (IWS), Guangzhou, China, 2019, pp. 1-3.

[16] B. Sanz-Izquierdo and E. A. Parker, "3D printed FSS arrays for long wavelength applications," The 8th European Conference on Antennas and Propagation (EuCAP 2014), The Hague, 2014, pp. 2382-2386.

[17] S. N. Azemi, K. Ghorbani and W. S. T. Rowe, "A reconfigurable FSS using a spring resonator element," IEEE Antennas and Wireless Propagation Letters, vol. 12, pp. 781-784, 2013.

[18] D. Ferreira, I. Cuiñas, R. F. S. Caldeirinha and T. R. Fernandes, "3-D mechanically tunable square slot FSS," IEEE Transactions on Antennas and Propagation, vol. 65, no. 1, pp. 242-250, Jan. 2017.

[19] D. F. Mamedes, A. Gomes Neto, J. C. e Silva and J. Bornemann, "Design of reconfigurable frequency-selective surfaces including the PIN diode threshold region," in IET Microwaves, Antennas \& Propagation, vol. 12, no. 9, pp. 1483-1486, 2018.

[20] Z. Lu, J. She and X. Yan, "A dual-band reconfigurable FSS composite structure based on MEMS switches," 2016 11th International Symposium on Antennas, Propagation and EM Theory (ISAPE), Guilin, 2016, pp. 630-632.

[21] A. G. Neto, J. C. e Silva, A. G. Barboza, D. F. Mamedes, I. B. G. Coutinho and M. de Oliveira Alencar, "Varactortunable four arms star bandstop FSS with a very simple bias circuit," 2019 13th European Conference on Antennas and Propagation (EuCAP), Krakow, Poland, 2019, pp. 1-5.

[22] A. Gomes Neto, J. C. e Silva, I. B. G. Coutinho, M. de O. Alencar, D. M. de Andrade, "Triple band reject frequency selective surface with application to $2.4 \mathrm{GHz}$ band," in Journal of Communication and Information Systems, vol. 35, no. 1, pp. 77-85, Apr. 2020.

[23] R. Simons, Coplanar Waveguide Circuits, Components, and Systems, USA: Wiley, 2001.

[24] http://www.hp.woodshot.com.

[25] https://www.everythingrf.com/rf-calculators/microstrip-calculator

[26] A. G. Neto, J. N. de Carvalho, A. N. da Silva, H. de Paiva Almeida Ferreira, I. S. S. Lima and J. I. Fernandes, "Four arms star: An useful geometry for switchable FSS," 2013 SBMO/IEEE MTT-S International Microwave \& Optoelectronics Conference (IMOC), Rio de Janeiro, Aug. 2013, pp. 1-5.

[27] Skyworks Solutions, Inc., Varactor_Diodes - Application Note, August 15, 2008.

[28] Skyworks Solutions, Inc., Varactor SPICE Models for RF VCO Applications - Application Note, August 5, 2015.

[29] Skyworks Solutions, Inc., SMV123x Series: Hyperabrupt Junction Tuning Varactors - Data Sheet, Note, July 28, 2016 